\title{
Lusioersily
}

\section{Encouraging children to think counterfactually enhances blocking in a causal learning task}

McCormack, T., Simms, V., McGourty, J., \& Beckers, T. (2013). Encouraging children to think counterfactually enhances blocking in a causal learning task. Quarterly Journal of Experimental Psychology, epub. https://doi.org/10.1080/17470218.2013.767847

Link to publication record in Ulster University Research Portal

\section{Published in:}

Quarterly Journal of Experimental Psychology

Publication Status:

Published (in print/issue): 01/02/2013

DOI:

10.1080/17470218.2013.767847

\section{Document Version}

Publisher's PDF, also known as Version of record

\section{General rights}

Copyright for the publications made accessible via Ulster University's Research Portal is retained by the author(s) and / or other copyright owners and it is a condition of accessing these publications that users recognise and abide by the legal requirements associated with these rights.

\section{Take down policy}

The Research Portal is Ulster University's institutional repository that provides access to Ulster's research outputs. Every effort has been made to ensure that content in the Research Portal does not infringe any person's rights, or applicable UK laws. If you discover content in the Research Portal that you believe breaches copyright or violates any law, please contact pure-support@ulster.ac.uk. 
This article was downloaded by: [109.148.101.194]

On: 21 February 2013, At: 07:36

Publisher: Routledge

Informa Ltd Registered in England and Wales Registered Number: 1072954 Registered office:

Mortimer House, 37-41 Mortimer Street, London W1T 3J H, UK

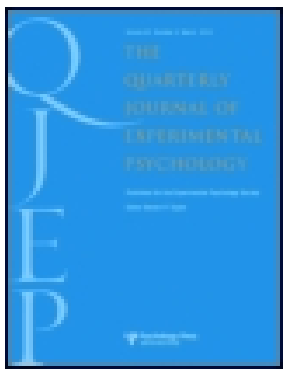

\title{
The Quarterly J ournal of Experimental Psychology
}

Publication details, including instructions for authors and subscription information:

http:// www.tandfonline.com/loi/ pqje20

Encouraging children to think counterfactually enhances blocking in a causal learning task

\author{
Teresa McCormack $^{a}$, Victoria Simms ${ }^{b}$, Jemma McGourty ${ }^{a} \&$ Tom Beckers ${ }^{c}$ \\ d \\ a School of Psychology, Queen's University Belfast, Belfast, UK \\ ${ }^{b}$ Department of Health Sciences, University of Leicester, Leicester, UK \\ c Department of Psychology, KU Leuven, Leuven, Belgium \\ ${ }^{d}$ Department of Clinical Psychology and Cognitive Science Center \\ Amsterdam, University of Amsterdam, Amsterdam, The Netherlands \\ Version of record first published: 21 Feb 2013.
}

To cite this article: Teresa McCormack , Victoria Simms, J emma McGourty \& Tom Beckers (2013): Encouraging children to think counterfactually enhances blocking in a causal learning task, The Quarterly J ournal of Experimental Psychology, DOI:10.1080/17470218.2013.767847

To link to this article: http:// dx.doi.org/ 10.1080/ 17470218.2013.767847

\section{PLEASE SCROLL DOWN FOR ARTICLE}

Full terms and conditions of use: http://www.tandfonline.com/page/terms-and-conditions

This article may be used for research, teaching, and private study purposes. Any substantial or systematic reproduction, redistribution, reselling, loan, sub-licensing, systematic supply, or distribution in any form to anyone is expressly forbidden.

The publisher does not give any warranty express or implied or make any representation that the contents will be complete or accurate or up to date. The accuracy of any instructions, formulae, and drug doses should be independently verified with primary sources. The publisher shall not be liable for any loss, actions, claims, proceedings, demand, or costs or damages whatsoever or howsoever caused arising directly or indirectly in connection with or arising out of the use of this material. 


\title{
Encouraging children to think counterfactually enhances blocking in a causal learning task
}

\author{
Teresa McCormack ${ }^{1}$, Victoria Simms ${ }^{2}$, Jemma McGourty ${ }^{1}$, and Tom Beckers ${ }^{3,4}$ \\ ${ }^{1}$ School of Psychology, Queen's University Belfast, Belfast, UK \\ ${ }^{2}$ Department of Health Sciences, University of Leicester, Leicester, UK \\ ${ }^{3}$ Department of Psychology, KU Leuven, Leuven, Belgium \\ ${ }^{4}$ Department of Clinical Psychology and Cognitive Science Center Amsterdam, University of Amsterdam, \\ Amsterdam, The Netherlands
}

\begin{abstract}
According to a higher order reasoning account, inferential reasoning processes underpin the widely observed cue competition effect of blocking in causal learning. The inference required for blocking has been described as modus tollens (if $\mathrm{p}$ then $\mathrm{q}$, not $\mathrm{q}$ therefore not $\mathrm{p}$ ). Young children are known to have difficulties with this type of inference, but research with adults suggests that this inference is easier if participants think counterfactually. In this study, 100 children (51 five-year-olds and 49 sixto seven-year-olds) were assigned to two types of pretraining groups. The counterfactual group observed demonstrations of cues paired with outcomes and answered questions about what the outcome would have been if the causal status of cues had been different, whereas the factual group answered factual questions about the same demonstrations. Children then completed a causal learning task. Counterfactual pretraining enhanced levels of blocking as well as modus tollens reasoning but only for the younger children. These findings provide new evidence for an important role for inferential reasoning in causal learning.
\end{abstract}

Keywords: Causal learning; Reasoning; Counterfactuals; Blocking.

There has been a wave of studies in the last decade that have suggested that inferential reasoning underpins some of the basic phenomena in causal learning that have been traditionally explained by associative theories of learning (De Houwer \& Beckers, 2003; De Houwer, Beckers, \& Vandorpe, 2005; Mitchell, Killedar, \& Lovibond, 2005; Vandorpe, De Houwer, \& Beckers, 2005). The findings of these studies have led some theorists to claim that human causal learning recruits higher order reasoning processes that operate over propositional knowledge, are effortful, and place demands on working memory (De Houwer,
2009; Mitchell, De Houwer, \& Lovibond, 2009). One potential, and novel, source of evidence for this type of account comes from research on children's causal learning. We have argued that, within a developmental context, it may be possible to characterize the reasoning processes necessary for particular types of causal learning and then demonstrate that children who, as yet, lack the ability to reason in this way show deficits in their causal learning as predicted by a higher order reasoning account (McCormack, Butterfill, Hoerl, \& Burns, 2009; McCormack, Simms, McGourty, \& Beckers, in press; Simms, McCormack, \&

Correspondence should be addressed to Teresa McCormack, School of Psychology, Queens University Belfast, Northern Ireland, UK, BT7 1NN. E-mail: t.mccormack@qub.ac.uk

This research was supported by a grant from the Economic and Social Research Council, UK, Grant RES-062-23-2109. 
Beckers, 2012). The aim of the current study was to add to this body of developmental evidence by showing that a manipulation that alters the likelihood of children reasoning in the necessary way also impacts on their causal learning.

One phenomenon that has been the focus of much of the relevant debate about the nature of causal learning is blocking. Blocking is widely observed in human and animal learning in many contexts (Dickinson, 2001; Dickinson, Shanks, \& Evenden, 1984; Kamin, 1968; Shanks, 1985). Demonstrations of blocking establish that being shown a cue $A$ that is paired with an outcome (denoted as $\mathrm{A}+$ ) seems to block new learning that a cue $B$ is causal, if $B$ is presented only along with $\mathrm{A}$ (in compound trials denoted as $\mathrm{AB}+$ ). When blocking is observed in human causal learning, lower causal ratings are given to $\mathrm{B}$ than to a control cue $\mathrm{C}$ from a pair $\mathrm{CD}+$ of which neither element has been presented on its own. Blocking has traditionally been explained by associative theories by assuming that establishing an association between $A$ and the outcome results in the outcome being already well predicted when the compound cue $\mathrm{AB}+$ is shown (Dickinson, 2001). Traditional associative theories assume that new learning only occurs when an outcome is surprising, as captured by the Rescorla-Wagner rule (Rescorla \& Wagner, 1972); because the outcome is already predicted through the presence of $\mathrm{A}$, new learning about $\mathrm{B}$ is blocked. Theories of causal learning have often been assessed on their ability to explain phenomena such as blocking and to account for the factors that influence it (De Houwer \& Beckers, 2002; Dickinson, 2001; Shanks, 2007, 2010). Indeed, one of the chief sources of evidence for the higher order reasoning account of causal learning is a series of findings that show that blocking is affected by a number of factors that cannot easily be explained by the associative approach (De Houwer et al., 2005).

One factor that has attracted considerable attention is that of additivity (Beckers, De Houwer, Pineño, \& Miller, 2005; Beckers, Miller, De Houwer, \& Urushihara, 2006; Haselgrove, 2010; Lovibond, Been, Mitchell, Bouton, \& Frohardt, 2003; Mitchell, Lovibond, \& Condoleon, 2005).
In studies manipulating additivity, participants are shown in an initial pretraining phase two causally efficacious cues (i.e., each yields an outcome, e.g., $\mathrm{A}+$ trials and $\mathrm{B}+$ trials). They are then shown either that if both causal cues occur together, the outcome is stronger than when cues occur by themselves (additive pretraining; the stronger outcome is denoted as ++$)$ or that if both causal cues occur together it results in an outcome of the same magnitude as a single cue (nonadditive pretraining). A considerable body of evidence now suggests that blocking is strongly affected by this manipulation: Blocking is robust following additive pretraining, but weak or absent following nonadditive pretraining (Beckers et al., 2005; Lovibond et al., 2003; Mitchell, Lovibond et al., 2005; Simms et al., 2012).

Under additive pretraining conditions, participants can engage in a chain of reasoning that leads to blocking: "A is causal and leads to outcome + . If $\mathrm{B}$ was also causal, the outcome for $\mathrm{AB}$ would be ++ . It is not, therefore $\mathrm{B}$ is not causal." As Mitchell, Lovibond et al. (2005) and Beckers et al. (2005) point out, this reasoning depends on making a modus tollens inference (if $p$ then $q$, not $q$ therefore not $p$ ). This inference is not possible under nonadditive pretraining conditions, because the outcome will be of the same magnitude regardless of whether or not $B$ is causal, and, according to the higher order reasoning account, blocking is therefore either weak or nonexistent if outcomes are not additive. This interpretation of Mitchell et al.'s findings, and those of other studies that have manipulated additivity, is controversial. One important reason for the controversy is that it has subsequently been demonstrated by Beckers et al. (2006) that blocking in rats can also be affected by additivity pretraining. Indeed, on the basis of this finding, Beckers et al. have claimed that rats are capable of inferential reasoning, arguing that associative models traditionally used to describe animal learning cannot account for the effect of additivity. Beckers et al.'s findings, and their interpretation, have been widely debated by comparative researchers (Castro \& Wasserman, 2009; Mitchell et al., 2009; Penn \& Povinelli, 2007; Shanks, 2010; Urcelay \& 
Miller, 2010), and there have been some recent attempts to explain their findings within the associative framework (Haselgrove, 2010; Schmajuk \& Larrauri, 2008) in order to resist the conclusion that rats must have sophisticated cognitive capacities.

The suggestion that the ability to make modus tollens inferences is necessary for blocking is particularly interesting from a cognitive developmental perspective, because it is well established that there are developmental changes in the ability to make this type of inference (Barrouillet, Grosset, \& Lecas, 2000; Braine \& Rumain, 1983). Using a child-friendly version of the well-known food allergy task, Simms et al. (2012) showed that additivity pretraining affected blocking in children aged 6-7 years, and not in a younger group of 4-to 5year-olds. Moreover, they found a correlation between modus tollens reasoning abilities and levels of blocking, and they also showed that only those children who were capable of making the relevant modus tollens inference demonstrated blocking on their task. This study provided the first evidence that making modus tollens inferences might be necessary for blocking under such circumstances. McCormack et al. (in press) replicated this finding of a relationship between modus tollens reasoning and blocking, and they also showed that, in addition to reasoning abilities, children's working memory abilities independently contributed to blocking.

The current study followed up these findings. As with any cross-sectional developmental correlational data, it could be argued that demonstrating a relationship between modus tollens reasoning abilities and blocking does not establish that blocking is causally related to, or a result of, such reasoning. Stronger evidence that blocking is a result of inferential reasoning would be in the form of a demonstration that intervening in and manipulating the likelihood that children successfully engaged in modus tollens reasoning also affected levels of blocking. However, the challenge is to find a manipulation that might affect the likelihood that children would successfully recruit such a reasoning process. When Mitchell, Lovibond et al. (2005) first described the reasoning they believed underpinned blocking, they described it as a counterfactual inference (see also Beckers et al., 2005), arguing that participants reason that if $\mathrm{B}$ had been causal, there would have been a strong outcome; there was not a strong outcome, therefore B is not causal. Strictly speaking, the necessary conditional inference need not necessarily be expressed subjunctively using counterfactual premisesparticipants can reach the correct conclusion if they reason with factual premises as expressed indicatively (e.g., if $\mathrm{B}$ was causal, there was a strong outcome ... ). Nevertheless, intuitively, it seems particularly plausible that participants will reach the correct conclusion if, when asked about B's causal status, they start to think counterfactually about what the outcome would have been if $\mathrm{B}$ had been causal.

In fact, this suggestion is not just intuitively plausible but can be justified on the basis of the empirical literature on conditional reasoning. While it is well known that even adults will sometimes make errors when asked to make modus tollens inferences (Manktelow, 2012), expressing conditional inferences in counterfactual rather than factual terms increases the likelihood that adults will correctly make modus tollens inferences (Byrne \& Tasso, 1999). For example, if adults are told that "If Linda had been in Dublin, then Cathy would have been in Galway", they are more likely to conclude, when told that Cathy had not been in Galway, that Linda had not been in Dublin than if they are given the factual premise "If Linda was in Dublin, Cathy was in Galway". Moreover, Thompson and Byrne (2002) have shown that if participants in a conditional reasoning task interpret premises in counterfactual rather than factual terms they are much more likely to make modus tollens inferences. Byrne and colleagues offer an interpretation of these findings in terms of mental models theory (Johnson-Laird \& Byrne, 1991). However, it is possible to remain agnostic about what theory of reasoning best explains these finding (see also Beckers et al., 2006) while exploiting it for our purpose of manipulating the likelihood that children will engage in modus tollens reasoning. 
In the current study, we varied whether children were encouraged to think factually or counterfactually about what they observed and then examined whether this impacted on levels of blocking. Our prediction was that encouraging children to think counterfactually would facilitate modus tollens reasoning, and thus blocking. We did this by varying the type of pretraining that children received before completing a causal learning task. Children were introduced to our child-friendly version of the food allergy task, in which children observe a toy robot being fed plastic foodstuffs. In this task, causally efficacious foods cause the robot to make a sound and his tummy to light up. Effects can be shown to be additive by demonstrating that two causally efficacious foods lead to a more intense outcome-more of the robot's tummy lighting up and a louder, higher, sound being produced. In the initial pretraining stage of the current study, all children received additivity pretraining - that is, they were shown that two causally efficacious cues in combination yielded a more intense outcome than when one causally efficacious cue was presented alone. Previously, Simms et al. (2012) found that only older 6-to 7-year-old children were affected by additivity pretraining, as indexed by its effect on blocking in causal learning. In the current study, we hoped to increase the likelihood that younger children of 5 years would use the information they had received about the additivity of causes and display blocking by encouraging them to think counterfactually about demonstrations involving pairs of cues.

Children were assigned to one of two pretraining groups for the second stage of pretraining: a factual pretraining group, in which children saw a series of demonstrations and simply answered factual questions about what had happened, or a counterfactual pretraining group, in which children saw the same demonstrations but were asked to think counterfactually about what they had seen. So, for example, during pretraining children were shown that A was causal (i.e., shown $\mathrm{A}+$ trials) and that $\mathrm{B}$ was not causal (B-trials). They then were shown $\mathrm{AB}+$ trials. In the factual group, children were asked what happens when $A$ and $B$ are fed to the robot together (a factual question). In the counterfactual group, children were asked to imagine that B had been causal and were then asked what the outcome would have been if that had been the case. The correct answer in this instance would have been to say that the strong rather than the weak outcome would have occurred.

Following these pretraining stages, children then completed a causal learning task using new sets of cues, and levels of blocking were measured. In addition to assessing blocking, we also gave children tasks designed to measure the contrasting cue competition effect of unovershadowing (Dickinson, 2001; Vandorpe \& De Houwer, 2005; although the term used to describe this effect varies between studies). In unovershadowing, participants see $\mathrm{A}$ - trials and are then shown $\mathrm{AB}+$ trials. Under such circumstances, participants are particularly likely to judge that B is causal, compared to circumstances in which they see a pair of cues $\mathrm{CD}+$, neither element of which is presented on its own. Simms et al. (2012) argued that unovershadowing requires participants to engage in the following chain of reasoning: "Either $\mathrm{A}$ or $\mathrm{B}$ must be causal. A is not causal, so B must be causal." This is a disjunctive inference that children find simpler than modus tollens, and Simms et al. showed that children were at ceiling in making such disjunctive inferences. Given this, we did not anticipate that the type of pretraining that children were given (factual or counterfactual) would have an impact on unovershadowing. Thus, our core prediction was that counterfactual pretraining would selectively increase levels of blocking. In addition to the core causal learning task, children were also asked separate sets of reasoning questions designed to measure modus tollens and disjunctive reasoning within the context of the main task. We predicted that children would find the disjunctive reasoning questions easy, but that performance on the modus tollens questions might be affected by the nature of pretraining.

\section{Method}

\section{Participants}

One hundred children took part in the study: fiftyone 5 -year-olds (mean age $=65$ months, range $=$ 
59 to 71 months) and forty-nine 6-7-year-olds (mean age $=78$ months, range $=72$ to 83 months). These children were recruited and tested individually in their schools. Testing took place over two separate testing sessions, each lasting around 25 minutes. Children from both age groups were randomly assigned to either a control $(N=49)$ or a counterfactual $(N=51)$ pretraining group.

\section{Apparatus and stimuli}

The causal learning task involved a purpose-built toy robot. The "tummy" of the robot had a transparent Perspex centre, and along both the right and left sides of the centre were two semiopaque light boxes that contained battery-powered LED lights. The bottom half of each light box was pink, and the top half was red. There was a hidden speaker inside the robot. There was a movable platform in the robot's mouth that toy foodstuffs could be placed on. When the robot's nose was pressed, the platform moved downwards into the robot's tummy and tilted, dropping any foodstuffs on it into the robot's tummy. After this one of three responses occurred: weak, strong, or no response. A weak response consisted of the bottom part of the robot's tummy lighting up along with a quiet, low noise. A strong response consisted of all of the robot's tummy lighting up, along with a loud, high noise. These responses lasted for $3 \mathrm{~s}$, following which the experimenter removed the foodstuff(s) from the robot's tummy through a hole at the back of the robot. The platform returned to the start position (the robot's mouth) automatically. The robot's responses were controlled by an input file selected by the experimenter from a computerized program on a laptop. There were six sets of 5 foods for use in the task itself, in addition to 16 foods used during the pretraining phases, 8 foods used for modus tollens and disjunctive reasoning testing, and 1 food item used to introduce the robot.

\section{Procedure}

The procedure consisted of three stages. In the first initial pretraining stage, the additivity of outcomes was demonstrated to children using a set of food cues that were not used in subsequent stages. In the second pretraining stage, children were shown further demonstrations involving pairs of cues and answered either factual or counterfactual questions concerning these cues, depending on which condition they were assigned to. Again, food items specific to this stage were used. The third phase was the learning stage in which children observed trials that they then answered test questions about. In this stage, there were three blocking and three unovershadowing tasks involving new cues, with children answering the test questions immediately after viewing the learning trials for each task. Because the procedure was lengthy (around 50 minutes in total), children completed three of these tasks in one session and three in a second session. Children received a mix of blocking and unovershadowing tasks in each session; they were randomly assigned to one of six different groups that each received the tasks in a different order. There was a reminder of the factual/counterfactual pretraining at the start of the second session. Both sessions occurred on the same day with a break of at least an hour between sessions.

Initial pretraining stage. Participants were introduced to the robot and were shown that pressing the robot's nose caused any foodstuffs placed on the platform to drop into his tummy. The additivity of outcomes was demonstrated using the series of trials shown in Table 1a. Each demonstration was repeated, and which foodstuff was used for each cue $(\mathrm{F}-\mathrm{I})$ was varied between participants. When a weak outcome occurred, the experimenter described the robot's responses as "a bit of his tummy lighting up", and when a strong outcome occurred, the experimenter described this as "all of his tummy lighting up”. In this part of pretraining, children were asked a series of comprehension questions in order to ensure that they understood the additivity of outcomes.

Factual and counterfactual pretraining. All children experienced four additional pretraining scenarios. These are displayed in Table $1 \mathrm{~b}$. Children were given two tasks where the outcome to the compound was weak and two tasks where the 
Table 1. Initial pretraining, factual/counterfactual pretraining, and learning phase

\begin{tabular}{llll}
\hline Stage & Type of task & Phase & \multicolumn{1}{c}{ Cues } \\
\hline a. Initial pretraining & & & $\mathrm{F}+/ \mathrm{G}-/ \mathrm{H}+/ \mathrm{I}-/ \mathrm{FG}+/ \mathrm{FH}++$ \\
b. Factual/counterfactual pretraining & Weak outcome & & $\mathrm{J}+/ \mathrm{K}-/ \mathrm{JK}+$ \\
& Strong outcome & & $\mathrm{L}+/ \mathrm{M}+/ \mathrm{LM}++$ \\
c. Learning stage & Blocking & Phase 1 & $\mathrm{~A}+, \mathrm{E}-$ \\
& & Phase 2 & $\mathrm{AB}+/ \mathrm{CD}+$ \\
& Unovershadowing & Phase 1 & $\mathrm{~A}-, \mathrm{E}+$ \\
& & Phase 2 & $\mathrm{AB}+/ \mathrm{CD}+$ \\
\hline
\end{tabular}

Note: - indicates no outcome, + indicates a weak outcome, ++ indicates a strong outcome. C items were controls; $\mathrm{E}$ items were fillers and ensured that there was at least one demonstration in which the outcome did not occur per trial. Trials in both pretraining stages were shown twice. Trials in each of the tasks of the learning stage were shown three times.

outcome to the compound was strong. The order of presentation of these scenarios was counterbalanced between children.

Counterfactual pretraining group. Weak outcome. After the child observed the two element and the compound trials the experimenter stated: "We know that food J makes part of the robot's tummy light up, and food $\mathrm{K}$ doesn't make the robot's tummy light up. So when we put food J and $\mathrm{K}$ into the robot's tummy together only part of the robot's tummy lit up." The experimenter then introduced the counterfactual component of the pretraining: "Now I want you to imagine something different. I want you to imagine that food $\mathrm{K}$ was a food that makes part of the robot's tummy light up as well. Ok, so imagine that both food J and food $\mathrm{K}$ were foods that make part of the robot's tummy light up by themselves. Ok, so are you imagining that both $\mathrm{J}$ and food $\mathrm{K}$ were foods that make part of the robot's tummy light up by themselves?" The child was then asked a counterfactual question: "What would have happened when we put both food $\mathrm{J}$ and food $\mathrm{K}$ in the robot's tummy together?" If the child answered that all the robot's tummy would have lit up, the experimenter confirmed verbally that they were correct. In a small minority of cases the child answered incorrectly that part of the robot's tummy would have lit up. When this occurred, the experimenter reminded children about the additivity of outcomes and then repeated the question a single time. Children were given two of these pretraining tasks.

Strong outcome. After the child observed the two elements and the compound trials the experimenter stated: "We know that both foods $\mathrm{L}$ and $\mathrm{M}$ make part of the robot's tummy light up on their own. So when we put both food $\mathrm{L}$ and $\mathrm{M}$ into the robot's tummy together they made all of the robot's tummy light up." Once again, the experimenter introduced the counterfactual component of the pretraining: "Now I want you to imagine something different. I want you to imagine that food L wasn't a food that makes part of the robot's tummy light up. Ok, so imagine that food L wasn't a food that makes part of the robot's tummy light up by itself, and food $\mathrm{M}$ was a food that makes part of the robot's tummy light up by itself . Ok, so are you imagining that L wasn't a food that makes part of the robot's tummy light up by itself, and food $\mathrm{M}$ was a food that makes part of the robot's tummy light up by itself?" The experimenter then asked the counterfactual question "What would have happened when we put both food $\mathrm{L}$ and food $\mathrm{M}$ in together?" If the child answered that part of the robot's tummy would have lit up, the experimenter confirmed verbally that they were correct. In a small minority of cases, the child answered incorrectly that all of the robot's tummy would have lit up. If this 
occurred, the experimenter reminded children about the additivity of outcomes and repeated the question once. Children were given two of these pretraining tasks.

Factual pretraining group. Children in the factual group observed identical pretraining trials to those for children in the counterfactual group, shown in Table 1b. The pretraining procedure for this group was identical up until the point at which children were asked questions about the cues. Rather than counterfactual questions, this group answered factual questions.

Weak outcome. After the child had observed the element and compound cues, the experimenter stated: "We know that food J makes part of the robot's tummy light up, and food K doesn't make the robot's tummy light up." The experimenter then asked the factual question: "What happens when we put both food $\mathrm{J}$ and $\mathrm{K}$ into the robot's tummy together?" Children never answered these questions incorrectly.

Strong outcome. After the child had observed the element and the compound cues, the experimenter stated: "We know that both foods L and M make part of the robot's tummy light up on their own." The experimenter then asked "What happens when we put both food $\mathrm{L}$ and $\mathrm{M}$ into the robot's tummy together?" Children never answered these questions incorrectly.

Learning stage. Learning trials immediately followed the two pretraining stages and followed the protocol in Table 1c. Unlike in tasks used with adults, blocking and unovershadowing were assessed on separate tasks to reduce the number of learning trials that children had to view before answering test questions. Children viewed the learning trials for each task as shown in Table 1c and then answered test questions. Note that for each task, the compound phase includes a pair of control cues, C and D. Neither of these cues was paired with the outcome on its own; responses to the experimental cue $\mathrm{B}$ were compared to responses to the control cue $\mathrm{C}$ to provide a measure of blocking or unovershadowing. Each participant completed six tasks: three blocking and three unovershadowing tasks. In each task, each trial shown in Table 1c was given three times (including the filler trials $\mathrm{E}-$ ), with the order of presentation of trials within each phase varied. Thus, for each task, participants observed 12 trials in total before being asked test questions (i.e., 72 learning trials altogether across the experiment), with the presentation order of blocking and unovershadowing tasks counterbalanced.

Each task used a new set of foodstuffs, and, within each set, foodstuffs were counterbalanced in terms of which element they represented (AD). Element $\mathrm{E}$ was a filler item that was included to ensure that there was at least one cue per blocking trial that was not paired with an outcome.

Test questions. Testing for each task followed immediately after its learning phases. Once children had observed all of the trials for any given task, the following questions were asked: "Is (food name B, e.g., cheese) a food that makes the robot's tummy light up?" and "Is (food name C, e.g., bread) a food that makes the robot's tummy light up?" The order of the questions (B or C) was counterbalanced, and children were required to give a yes or no response. Children were also asked a forced-choice question: "If you had to choose one of these foods to make the robot's tummy light up, which one would you choose (experimenter holds out B and C)?" Children could either name or point to one of the foods (control or experimental). No feedback was given to participants.

In the first testing session, children completed three cue competition tasks and two reasoning questions (see below). In the second testing session, they were given a brief reminder of the additivity pretraining, and then factual or counterfactual pretraining was refreshed by giving children two tasks identical to those used in pretraining in the first session (one with a weak outcome and one with a strong outcome). After this, children completed the remaining three cue competition tasks and answered the final two reasoning questions. 
Reasoning questions. All children also answered two disjunctive and two modus tollens reasoning questions related to the operation of the robot, with new foods used for each question.

Modus tollens questions. Children initially observed that a compound of two novel foods made part of the robot's tummy light up a single time. The experimenter then stated: "Look, when we put food $\mathrm{N}$ and $\mathrm{O}$ in the robot's tummy together part of the robot's tummy lights up." The experimenter then asked: "Do both of the foods (hold up $\mathrm{N}$ and O) or only one of the foods make the robot's tummy light up?" Answering this question requires children to reason that if both foods are causal, the whole of the robot's tummy would light; it did not, so both cannot be causal (a modus tollens inference).

Disjunctive questions. Children initially observed that a compound of two novel foods made part of the robot's tummy light up a single time. The experimenter stated: "Look when we put food $\mathrm{P}$ and $\mathrm{Q}$ in the robot's tummy together part of the robot's tummy lights up." The experimenter then asked: "One of these foods (hold up P and Q) doesn't make the robot's tummy light up. Does the other food make the robot's tummy light up?" Answering this question involves reasoning that because either $\mathrm{P}$ or $\mathrm{Q}$ must be causal, and one of them is not causal, the other food must be causal (a disjunctive inference).

\section{Results}

During the counterfactual/factual pretraining session, children were asked four questions in total. These questions were always correctly answered in the factual group. In the counterfactual group, if children gave the wrong answer they were reminded about the additivity of outcomes and asked the question a second time. To examine performance on these counterfactual pretraining questions, we gave children a score of 2 points if they answered a question correctly the first time (maximum score of 8) and 1 point if they answered it correctly when it was repeated. Although we anticipated that children might find the counterfactual questions difficult, children scored on average 7 out of 8 (i.e., most questions were answered correctly first time). There were no significant relationships between how accurate children were on the counterfactual questions and subsequent performance, and therefore no further analyses of these data will be reported.

The presence of cue competition effects in children's causal learning was assessed in two ways: through comparing numbers of "yes" responses to experimental versus control cues, and by examining how often children chose the experimental cue versus the control cue when asked to choose a cue to make the robot light up.

\section{Choice scores}

Children chose between the experimental cue and the control cue three times for each task. Choice scores were calculated as follows: Children were given one point each time they chose the control
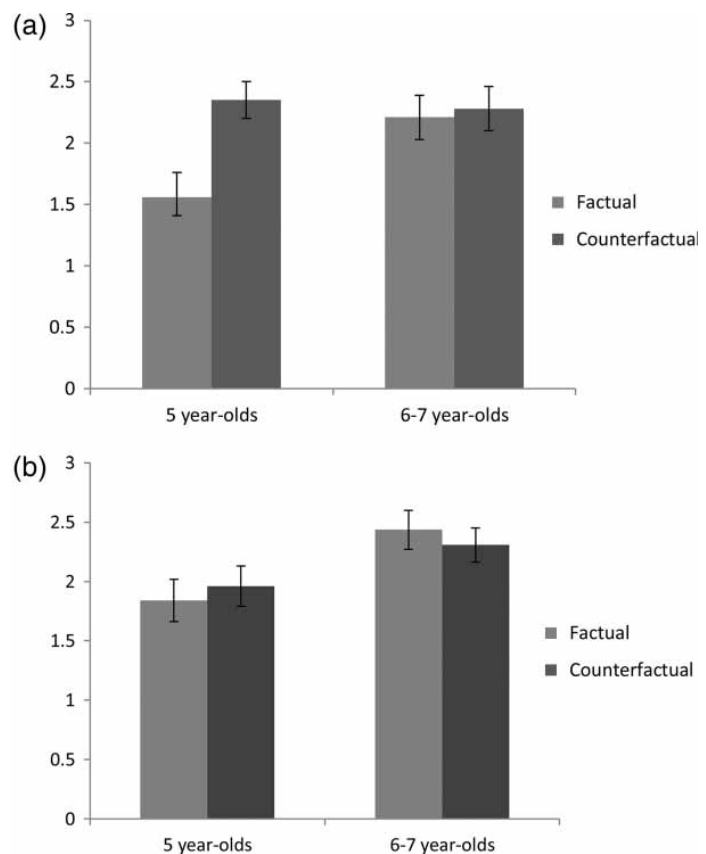

Figure 1. (a) Blocking choice scores as a function of pretraining and age group; maximum score is 3 ( $\pm 1 \mathrm{SE}$ ). (b) Unovershadowing choice scores as a function of age and pretraining type; maximum score is 3 ( $\pm 1 \mathrm{SE})$. 
cue in blocking tasks, whereas in unovershadowing tasks they were given one point each time they chose the experimental cue. Figures $1 \mathrm{a}$ and $1 \mathrm{~b}$ show children's scores as a function of age and pretraining type. It can be seen from the figures that pretraining seems to have selectively impacted on the younger children's blocking choice scores. Initial analyses examined whether the levels of blocking and overshadowing that were obtained in each group were significantly greater than that expected by chance, using one-sample $t$ tests (test value $=1.5$ ). Blocking was not significant for the 5 -year-olds in the factual pretraining group, $t(24)=0.30, p=.77$, and unovershadowing was only marginally significant in this group, $t(24)=$ 1.89, $p=.07$. However, significant blocking, $t(24)=5.79, \quad p<.001$, and unovershadowing, $t(24)=2.72, p<.02$, were observed for this age group in the counterfactual pretraining group. For the older 6-to 7-year-old group, significant blocking and unovershadowing were observed in both pretraining groups, all $t \mathrm{~s}(23)>3.9, \quad p s<.01$. Further analyses examined whether pretraining had a significant impact on choice scores. An initial repeated measures analysis of variance (ANOVA) on choice scores, with a between-subjects factor of type of pretraining (factual versus counterfactual) and a within-subjects factor of task (blocking versus overshadowing) found a two-way interaction between task and pretraining, $F(1,98)=5.24, p<.03$. Therefore, subsequent analyses examined blocking and unovershadowing separately.

Blocking. A two-way ANOVA on blocking choice scores with between-subjects factors of age and pretraining found a main effect of pretraining, $F(1$, $96)=5.88, p<.02$, and also a significant interaction between age and pretraining, $F(1,96)=$ $4.08, p<.05$. Further post hoc analyses showed that the effect of pretraining was significant for the younger group only, $t(49)=-3.19, p<.005$, with children in the counterfactual pretraining group having higher blocking choice scores. Moreover, the effect of age was significant for the factual pretraining group, $t(47)=-2.40, p<.03$, but not for the counterfactual pretraining group, $t(49)=0.29, p=.77$.

Unovershadowing. A two-way ANOVA on unovershadowing choice scores with between-subjects factors of age and pretraining found a main effect of age, $F(1,96)=8.06, p<.01$, with older children having higher choice scores, but no significant effect of pretraining and no significant interaction between age and pretraining, both $F_{\mathrm{S}}<1$.

\section{Yes responses}

Figure $2 \mathrm{a}$ shows the average number of "yes" responses given to experimental and control cues in the blocking task as a function of age group and pretraining; these vary from $0-3$ because children received each task three times. Figure $2 \mathrm{~b}$ shows the average number of positive responses to
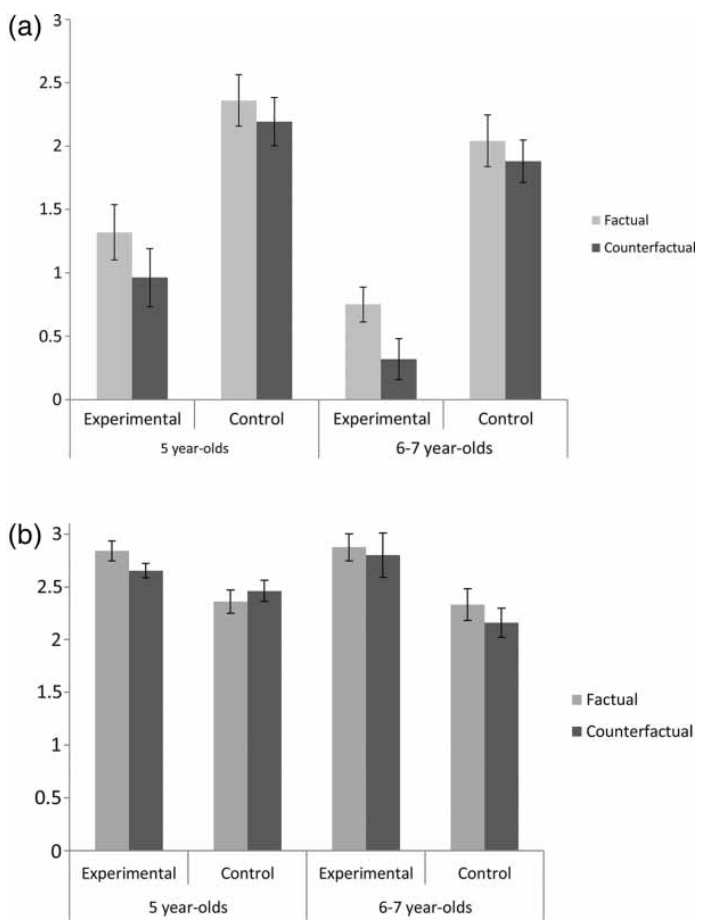

Figure 2. (a) Number of "yes" responses in blocking tasks to experimental and control cues as a function of pretraining type and age ( $\pm 1 \mathrm{SE})$. (b) Number of "yes" responses in unovershadowing tasks to experimental and control cues as a function of pretraining and age group ( $\pm 1 \mathrm{SE})$. 
experimental and control cues in the unovershadowing task as a function of age group and pretraining.

Blocking. A three-way ANOVA was conducted on the number of responses to each cue type in the blocking tasks, with a within-subjects factor of cue type (control versus experimental) and between-subjects factors of pretraining (factual versus counterfactual) and age group (5-year-olds versus 6- to 7-year-olds). The main effect of cue type was significant, $F(1,96)=125.60$, $p<.001$, with fewer positive responses to experimental than to control cues. This indicates that blocking was at a significant level for the sample as a whole. The main effect of age group was also significant, $F(1,96)=8.86, p<.01$, with older children giving fewer positive responses than younger children. The main effect of pretraining was also significant, $F(1,96)=4.13$, $p<.05$, with fewer positive responses following counterfactual pretraining. There were no significant interactions. Our prediction had been that counterfactual pretraining would increase blocking, manifested as a reduction in the number of positive responses given to experimental cues. Counterfactual pretraining did have an impact on children's responses, but its effect was to reduce overall levels of positive responses to both experimental and control cues.

Unovershadowing. A three-way ANOVA was conducted on the number of responses to each cue type in the unovershadowing tasks, with a within-subjects factor of cue type (control versus experimental) and between-subjects factors of pretraining (factual versus counterfactual) and age group (5-year-olds versus 6- to 7-year-olds). The main effect of cue type was significant, $F(1,96)=34.35, p<.001$, indicating that unovershadowing was at a significant level for the sample as a whole. However, there were no other significant main effects and no significant interactions. Hence, levels of overshadowing were not affected by age or by pretraining.

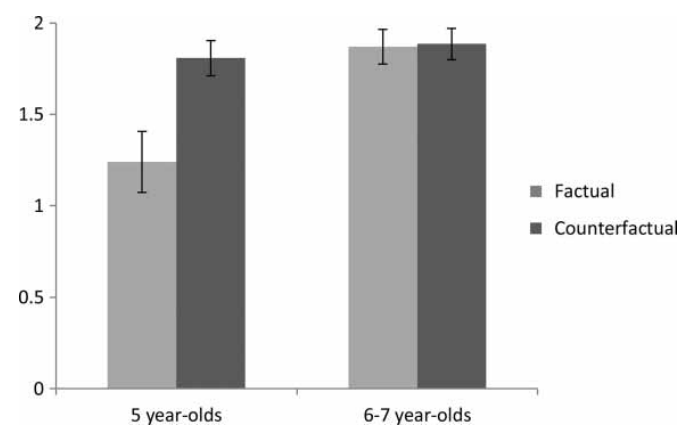

Figure 3. Number of modus tollens questions correct as a function of age and pretraining $( \pm 1 \mathrm{SE})$.

\section{Performance on reasoning questions}

The final analyses focused on children's reasoning scores. These scores varied from 0-2. Children were at ceiling on disjunctive reasoning questions, with a mean score of 1.9 . Figure 3 shows modus tollens reasoning scores as a function of age and pretraining. The graph shows that pretraining enhanced modus tollens reasoning in the younger group only. A two-way ANOVA on modus tollens reasoning scores with between-subjects factors of age and pretraining found a significant effect of age, $F(1,96)=9.41, p<.005$, a significant effect of pretraining, $F(1,96)=6.17$, $p<.02$, and a significant interaction between age and pretraining, $F(1,96)=5.96, p<.02$. Post hoc analysis showed that the effect of pretraining was significant for the younger group only, $t(49)=-2.98, p<.005$. Moreover, the effect of age was significant for the factual pretraining group, $t(47)=-3.31, p<.005$, but not significant for the counterfactual pretraining group, $t(49)=-0.55, p=.58$. Further analyses examined whether there was any relationship between children's performance on the modus tollens reasoning questions and levels of blocking. Analysis using Pearson's correlations revealed a significant correlation between blocking choice scores and modus tollens scores, $r(100)=.278, p<.01$, and moreover that the partial correlation between blocking choice scores and reasoning scores remained significant when controlling for chronological age, $r(100)=.243, p<.02$. 


\section{Discussion}

Our prediction-that encouraging children to think counterfactually would enhance modus tollens reasoning and blocking - was confirmed for the 5year-old group in this study. Five-year-olds who received counterfactual pretraining performed better on the modus tollens questions than children who received factual pretraining, and children of this age performed at a level equivalent to 6- to 7year-olds on these reasoning questions following counterfactual pretraining. Moreover, levels of blocking, as measured by choice scores, were significantly higher in the 5-year-olds who received counterfactual pretraining. Indeed, when 5-year-old children received counterfactual pretraining they showed blocking at a level equivalent to that of older children, whereas there were age differences in the absence of this pretraining. Counterfactual pretraining did not affect the performance of the older group either on the modus tollens reasoning questions or on blocking, but this is likely to be because this group was already performing well even following factual pretraining.

Replicating Simms et al. (2012) and McCormack et al. (in press), levels of blocking were also correlated with modus tollens reasoning abilities. These findings are the first that we are aware of that show that children who are encouraged to think counterfactually will be more likely to successfully make modus tollens inferences. Moreover, this study is the first with either children or adults to establish that participants who are trained to think counterfactually will show higher levels of blocking. The findings strongly suggest that reasoning abilities-specifically, the ability to make modus tollens inferences-underpin the blocking effect in this type of task. Thus, they provide a new type of evidence for a higher order reasoning account (De Houwer, 2009; De Houwer et al., 2005) of this important cue competition effect.

\section{Measures of blocking}

The pretraining effect for the 5-year-olds was in line with predictions for one measure of blocking: the number of times participants chose a control rather than experimental cue as one that would be causally efficacious. Beckers, Vandorpe, Debeys, and De Houwer (2009) have previously argued that because, unlike adults, children are not asked to rate the causal efficacy of cues on a scale, this forced-choice measure provides the best assessment of cue competition effects such as blocking in children (but see Simms et al., 2012). However, blocking was also assessed by comparing the number of "yes" responses to control cues versus experimental cues given by participants when asked whether a cue was causal. On this measure, pretraining children to think counterfactually did have an impact on responses, but in fact its impact was to reduce the overall number of positive responses that participants made, rather than selectively reducing the number of positive responses to the experimental (noncausal) cue.

A straightforward explanation of this generalized pretraining effect is possible, because modus tollens reasoning could also affect the number of times that children gave positive responses to the control cue (Simms et al., 2012). This is because, for any given control cue $\mathrm{C}$, children will have seen that cue only along with another cue $\mathrm{D}$ (i.e., the $\mathrm{CD}+$ trials). Children can reason that if both $\mathrm{C}$ and $\mathrm{D}$ were causal, there would have been a strong outcome and, using modus tollens reasoning, conclude that only one of these cues can be causal. Thus, modus tollens reasoning could potentially reduce the number of positive responses to the control cue $\mathrm{C}$ as well as to the experimental cue $\mathrm{B}$-the pattern observed in our data. Evidence for this suggestion comes from the fact that there was a significant negative correlation between modus tollens scores and the number of times that children gave "yes" responses to $\mathrm{C}$ cues $(r=-.229, p<.03)$ as well as $\mathrm{B}$ cues $(r=-.418, p<.001)$ in blocking tasks.

Thus, the fact that counterfactual training reduces the overall number of positive responses to both control and experimental cues in the blocking task is entirely consistent with our argument that counterfactual training enhances modus tollens reasoning. Why, though, does counterfactual training selectively increase the likelihood that participants choose the control cue over the experimental cue when forced to choose one as causal? The answer to this question lies in the 
differential impact that modus tollens reasoning should have on children's beliefs that a cue cannot be causal. Modus tollens reasoning will completely disambiguate the status of the experimental cues in blocking tasks, because it licenses the conclusion that the experimental cue $\mathrm{B}$ cannot be causal (since $\mathrm{A}$ is the causal cue). However, with regard to the control cues, the same reasoning only licenses the conclusion that $\mathrm{C}$ and $\mathrm{D}$ cannot both be causal - it remains possible that $\mathrm{C}$ rather than $\mathrm{D}$ is in fact the causal cue. The effect of this seems to be to reduce the likelihood that $\mathrm{C}$ is judged as being causal when children are asked to give a yes/no answer, but to nevertheless allow children to conclude that $\mathrm{C}$ is more likely to be causal than $\mathrm{B}$ when forced to choose between the two cues.

We note, though, that this account of the data would suggest that the number of positive responses to control cues should also be affected by counterfactual pretraining in the unovershadowing task, and this was not the case (see Figure 2b). It is possible that, following counterfactual pretraining, children may be more likely to engage in modus tollens reasoning regarding control cues in the blocking tasks because this type of reasoning disambiguates the status of experimental cues in those tasks. If children are thinking counterfactually about outcomes regarding experimental cues in the blocking task and then reasoning using modus tollens, they may be likely to extend this reasoning to control cues. By contrast, in the unovershadowing task, children need only engage in simpler disjunctive reasoning in order to disambiguate the status of the experimental cues, which children of both ages found easy and which would not be facilitated by counterfactual thinking. Given this, they may have been less inclined to think counterfactually about the outcomes paired with the control cues in unovershadowing tasks, explaining the lack of a pretraining effect on the numbers of positive responses to these cues in the unovershadowing task.

Why does counterfactual pretraining affect blocking? The fact that pretraining did not affect performance on the unovershadowing task allows us to rule out some simpler alternative explanations of why the pretraining manipulation was effective. It suggests that the effect of encouraging children to think counterfactually was not simply to increase the extent to which children engaged with the task or remembered the trials. We interpret the findings as indicating that counterfactual pretraining affects the likelihood that children reason appropriately at test about the status of cues using the additivity information about causes that they had been shown in the first stage of training. However, it might be argued that the counterfactual pretraining had a more direct effect on performance by actually providing children with more opportunity to learn that causes were additive. In designing our task, we aimed to match the amount of information that demonstrated additivity across the factual and counterfactual pretraining groups. Nevertheless, we need to consider whether the counterfactual pretraining condition did provide children with additional information about the additivity of causes. In the counterfactual pretraining condition, children had to make judgements about the combined effects of cue types that had not actually been demonstrated to them (e.g., children in this condition had to imagine that the causal status of a cue $\mathrm{K}$, previously shown not to be causal, had been different and then judge what the result of feeding JK to the robot would have been). Children were given verbal feedback on their answers, and it could be argued that this helped children realize that the additivity rule generalized to a broader range of cues than it did for children in the factual group (e.g., applied not just to a situation in which $\mathrm{K}$ was noncausal, as had been demonstrated to both groups, but also to a situation in which $\mathrm{K}$ was causal, which only the counterfactual group imagined).

An important reason for doubting this interpretation is that children's performance on the counterfactual questions indicated that they did not have difficulties generalizing this rule. Children had to use the additivity rule to answer the counterfactual questions themselves correctly, and they did so for the majority of these questions. This shows that they were not conservative about using the additivity rule. It is true that on the minority of occasions in which children in this group gave the incorrect 
answer, they were explicitly reminded of the additivity rule (children were never reminded of the rule in the factual training condition because they always gave the correct answers). However, there was no relationship between how often children received this reminder and their subsequent performance on the main task. Thus, we would interpret the effect of the counterfactual training as assisting children to effectively apply at test the rule about the additivity of causes that they had previously learned in the first stage of pretraining rather than providing additional information about the additivity of causes.

Our finding that counterfactual training affected blocking has important implications for how the effect of additivity pretraining itself on blocking is interpreted in previous studies (Beckers et al., 2005; Lovibond et al., 2003; Mitchell, Lovibond et al., 2005). In particular, it seems difficult to see how the effect of encouraging children to think counterfactually on blocking could be accounted for within an associative explanation of additivity, such as that of Haselgrove (2010). Hence, we would argue that explaining additivity effects remains an important challenge to any account of blocking that does not appeal to a role for reasoning processes in causal learning (Mitchell et al., 2009).

\section{Reasoning and counterfactual thinking}

How do our findings link with the broader literature on reasoning and its development? There are three strands of empirical work on reasoning that link with our study, although in each case there are important differences between these bodies of work and our methodology. First, there are some studies that suggest that encouraging children to think counterfactually has an impact on their reasoning (e.g., Amsel, Trionfi, \& Campbell, 2005; Dias \& Harris, 1988, 1990; Leevers \& Harris, 2000). However, these specific studies require children to reason syllogistically using "false" premises (e.g., "Fish live in trees. Freddy is a fish. Does Freddy the Fish live in a tree?"). The findings from these studies indicate that getting children to set aside their beliefs about the real world and imagine that things were different seems to facilitate accurate deductions. Typically children in these studies are encouraged to think counterfactually in the broad sense of being encouraged to make-believe or pretend that the world is different (Dias \& Harris, 1988, 1990), before being asked to reason syllogistically.

Although, arguably, such studies also demonstrate that counterfactual thinking impacts on children's reasoning, the methodology used in those studies was very different to that used in the present study, in which the premises that children had to reason with were true but children were asked to imagine alternative outcomes to those they had observed. The second strand of research that links to our findings, and indeed helped motivated our study, is that of Byrne and colleagues (Byrne \& Tasso, 1999; Thompson \& Byrne, 2002) who found that counterfactual thinking in adults facilitated modus tollens reasoning. However, our study differs from those of Byrne, which involved varying whether premises in a conditional reasoning task were expressed factually or counterfactually and then asking participants explicitly to reason using the premises. In our study, children were encouraged to think counterfactually only in a pretraining phase, and this pretraining had carry-over effects in terms of impacting on their performance even on a task in which conditional reasoning was not directly assessed. In this respect, our pretraining manipulation has commonalities with a third set of studies that have encouraged participants to adopt what has been described as a "counterfactual mind-set" (Epstude \& Roese, 2008; Kray \& Galinsky, 2003; Markman, Lindberg, Kray, \& Galinsky, 2007; Nestler \& von Collani, 2008) in advance of completing another cognitive task. However, we note that the effect of counterfactual pretraining that we have demonstrated is considerably narrower than effects demonstrated in counterfactual priming studies, in which initially encouraging participants to think counterfactually has an effect on performance on completely unrelated reasoning tasks.

The authors of such studies have argued that adopting a counterfactual mind-set may encourage participants to think more broadly and flexibly about evidence that they are presented with 
(Epstude \& Roese, 2008; Markman et al., 2007) or to consider more carefully the relationships between stimuli (what Kray, Galinsky, \& Wong, 2006, refer to as adopting a relational processing style). Because the task affected by our counterfactual pretraining involved making inferences concerning the same type of content that children had reasoned about during pretraining, we do not interpret our findings as indicating a global change in processing style. Nevertheless, the success of our manipulation suggests that it would be interesting to explore whether encouraging children to think counterfactually has wider effects on their performance on other cognitive tasks.

\section{Counterfactual thinking and causal cognition}

We conclude by considering how our findings relate to long-standing questions about the nature of the relationship between counterfactual and causal cognition. The idea that counterfactual thinking can lead to participants considering nonactual possibilities, thus impacting on their causal judgements, has been extensively discussed within the literature on causal cognition (Harris, German, \& Mills, 1996; Hoerl, McCormack, \& Beck, 2011; Mandel, 2003; Spellman, Kincannon, \& Stose, 2005; Wells \& Gavanski, 1989). This suggestion derives from philosophical accounts of the nature of causation that tightly link causal and counterfactual judgements (Hart \& Honoré, 1985; Mackie, 1974). Put simply, the philosophical idea is that what it is to say that event $\mathrm{X}$ caused event $\mathrm{Y}$ is to say that if $\mathrm{X}$ had not happened, then $\mathrm{Y}$ would not have occurred. Translated into a psychological principle, the claim is that, when deciding whether an outcome was caused by a particular event, one needs to think counterfactually about whether the outcome would have occurred in the absence of that event (see Harris et al., 1996, for a developmental version of this claim). If the outcome would not have occurred without the event, then the event is judged to be causal (what Spellman et al., 2005, refer to as the "but for" test). Although we have found that counterfactual thinking has an effect on children's causal judgements, it is important to stress that the role of counterfactual thinking that we are highlighting is not that implied by such an approach (Hoerl et al., 2011). That is, when children are assessing the status of experimental cues, we are not assuming that they are using counterfactual thinking to establish whether or not the outcome would have occurred in the absence of the cue (i.e., they are not applying the "but for" test). Rather, on blocking tasks, counterfactual thinking serves to highlight that, given the additive relationship between cues and the outcome, which can be captured in the form of a conditional rule, $\mathrm{B}$ cannot be causal. Thus, although we are arguing that counterfactual thinking can form part of the process involved in making causal judgements in this type of task, we are not interpreting our results as evidence for a counterfactual theory of causal judgement such as that described by Harris et al. (1996). Rather, our results provide an interesting addition to the literature on the relationship between counterfactual and causal cognition, because they suggest that counterfactual cognition may make a quite specific type of contribution to causal learning: by facilitating the extensively studied phenomenon of blocking (for a more substantial discussion of this point, see McCormack, Frosch, \& Burns, 2011).

Unpacking the contribution that counterfactual thinking makes to causal cognition is particularly important from a developmental perspective, because it can help characterize the nature of the cognitive changes that are important for the development of causal learning (Corrigan \& Denton, 1996; Schlottmann, 2001; Simms et al., 2012; Zimmerman, 2000). The key finding in this context is that counterfactual pretraining abolished age differences in levels of blocking, at least as measured by choice scores. This finding suggests that age differences in blocking reported previously (McCormack et al., 2009; McCormack et al., in press; Simms et al., 2012) may be at least in part due to developmental improvements in reasoning abilities, because when young children's reasoning was facilitated through counterfactual pretraining, age effects were no longer significant. More specifically, they indicate that one potential source of these improvements may be that older children are more likely to spontaneously think 
counterfactually about evidence they are presented with. Thus, our findings imply a novel way in which the development of counterfactual thinking could contribute to the development of causal cognition.

Original manuscript received 28 March 2012 Accepted revision received 4 September 2012 First published 19 February 2013

\section{REFERENCES}

Amsel, E., Trionfi, G., \& Campbell, R. (2005). Reasoning about make-believe and hypothetical suppositions: Towards a theory of belief-contravening reasoning. Cognitive Development, 20, 545-575.

Barrouillet, P., Grosset, N., \& Lecas, J. (2000). Conditional reasoning by mental models: Chronometric and developmental evidence. Cognition, 75, 237-266.

Beckers, T., De Houwer, J., Pineño, O., \& Miller, R. R. (2005). Outcome additivity and outcome maximality influence cue competition in human causal learning. Journal of Experimental Psychology: Learning, Memory, and Cognition, 31, 238-249.

Beckers, T., Miller, R. R., De Houwer, J., \& Urushihara, K. (2006). Reasoning rats: Forward blocking in Pavlovian animal conditioning is sensitive to constraints of causal inference. Journal of Experimental Psychology: General, 135, 92-102.

Beckers, T., Vandorpe, S., Debeys, I., \& De Houwer, J. (2009). Three-year-olds' retrospective revaluation in the blicket detector task. Backward blocking or recovery from overshadowing? Experimental Psychology, 56, $27-32$.

Braine, M. D. S., \& Rumain, B. (1983). Logical reasoning. In J. H. Flavell \& E. M. Markman (Eds.), Handbook of child psychology: Vol. 3. Cognitive development (pp. 263-340). New York, NY: Wiley and Sons.

Byrne, R. M. J., \& Tasso, A. (1999). Deductive reasoning with factual, possible, and counterfactual conditionals. Memory \& Cognition, 27, 724-740.

Castro, L., \&Wasserman, E. A. (2009). Rats and infants as propositional reasoners: A plausible possibility? Behavioral and Brain Sciences, 32, 203-204.
Corrigan, R., \& Denton, P. (1996). Causal understanding as a developmental primitive. Developmental Review, 16, 162-202.

De Houwer, J. (2009). The propositional approach to associative learning as an alternative for association formation models. Learning \& Behavior, 37, 1-20.

De Houwer, J., \& Beckers, T. (2002). A review of recent developments in research and theories on human contingency learning. Quarterly Journal of Experimental Psychology, 55B, 289-310.

De Houwer, J., \& Beckers, T. (2003). Secondary task difficulty modulates forward blocking in human contingency learning. Quarterly Journal of Experimental Psychology, 54B, 345-357.

De Houwer, J., Beckers, T., \& Vandorpe, S. (2005). Evidence for the role of higher order reasoning processes in cue competition and other learning phenomena. Learning \& Behavior, 33, 239-249.

Dias, M. G., \& Harris, P. L. (1988). The effect of makebelieve play on deductive reasoning. British Journal of Developmental Psychology, 6, 207-221.

Dias, M. G., \& Harris, P. L. (1990). The influence of the imagination on reasoning by young-children. British Journal of Developmental Psychology, 8, 305-318.

Dickinson, A. (2001). Causal learning: An associative analysis. Quarterly Journal of Experimental Psychology, 49B, 60-80.

Dickinson, A., Shanks, D. R., \& Evenden, J. L. (1984). Judgment of act-outcome contingency: The role of selective attribution. Quarterly Journal of Experimental Psychology, 36A, 29-50.

Epstude, K., \& Roese, N. J. (2008). The functional theory of counterfactual thinking. Personality and Social Psychology Review, 12, 168-192.

Harris, P. L., German, T., \& Mills, P. (1996). Children's use of counterfactual thinking in causal reasoning. Cognition, 61, 233-259.

Hart, H. L. A., \& Honoré, T. (1985). Causation in the law. Oxford, UK: Clarendon Press.

Haselgrove, M. (2010). Reasoning rats or associative animals? A common-element analysis of the effects of additive and subadditive pretraining on blocking. Journal of Experimental Psychology: Animal Behavior Processes, 36, 296-306.

Hoerl, C., McCormack, T., \& Beck, S. (2011). Understanding counterfactuals, understanding causation. Oxford, UK: Oxford University Press.

Johnson-Laird, P. N., \& Byrne, R. M. J. (1991). Deduction. Hove, UK: Psychology Press.

Kamin, L. J. (1968). Attention-like processes in classical conditioning. In M. R. Jones (Ed.), Miami 
Symposium on the Prediction of Behavior: Aversive Stimulation (pp. 9-32). Coral Gables, FL: University of Miami Press.

Kray, L. J., \& Galinsky, A. D. (2003). The debiasing effect of counterfactual mind-sets: Increasing the search for disconfirmatory information in group decisions. Organizational Behavior and Human Decision Processes, 91, 69-81.

Kray, L. J., Galinsky, A. D., \& Wong, E. (2006). Thinking within the box. The relational processing style elicited by counterfactual mind-sets. Journal of Personality and Social Psychology, 91, 33-48.

Leevers, H., \& Harris, P. L. (2000). Counterfactual syllogistic reasoning in normal 4-year-olds, children with learning disabilities, and children with autism. Journal of Experimental Child Psychology, 76, 64-87.

Lovibond, P. F., Been, S., Mitchell, C. J., Bouton, M. E., \& Frohardt, R. (2003). Forward and backward blocking of causal judgment is enhanced by additivity of effect magnitude. Memory $\xi^{\circ}$ Cognition, 31, 133-142.

Mackie, J. L. (1974). The cement of the universe: A study of causation. Oxford, UK: Oxford University Press.

Mandel, D. (2003). Judgment dissociation theory: An analysis of differences in causal, counterfactual, and covariational reasoning. Journal of Experimental Psychology: General, 132, 419-434.

Manktelow, K. (2012). Thinking and reasoning: An introduction to the psychology of reason, judgment, and decision making. Hove, UK: Psychology Press.

Markman, K. D., Lindberg, M. J., Kray, L. J., \& Galinsky, A. D. (2007). Implications of counterfactual structure for creative generation and analytical problem solving. Personality and Social Psychology Bulletin, 33, 312-324.

McCormack, T., Butterfill, S., Hoerl, C., \& Burns, P. (2009). Cue competition effects and young children's causal and counterfactual inferences. Developmental Psychology, 45, 1563-1575.

McCormack, T., Frosch, C., \& Burns, P. (2011). The relationship between children's causal and counterfactual judgments. In C. Hoerl, T. McCormack, \& S. R. Beck (Eds.), Understanding counterfactuals, understanding causation: Issues in philosophy and psychology (pp. 54-74). Oxford, UK: Oxford University Press.

McCormack, T., Simms, V., McGourty, J., \& Beckers, T. (in press). Blocking in children's causal learning depends on working memory and reasoning abilities. Journal of Experimental Child Psychology.

Mitchell, C. J., De Houwer, J., \& Lovibond, P. F. (2009). The propositional nature of human associative learning. Behavioral and Brain Sciences, 32, 183-198.

Mitchell, C. J., Killedar, A., \& Lovibond, P. F. (2005). Inference-based retrospective revaluation in human causal judgments requires knowledge of withincompound relationships. Journal of Experimental Psychology: Animal Behavior Processes, 31, 418-424.

Mitchell, C. J., Lovibond, P. F., \& Condoleon, M. (2005). Evidence for deductive reasoning in blocking of causal judgments. Learning and Motivation, 36, 77-87.

Nestler, S., \& von Collani, G. (2008). Hindsight bias and the activation of counterfactual mind-sets. Experimental Psychology, 55, 342-349.

Penn, D. C., \& Povinelli, D. J. (2007). Causal cognition in human and nonhuman animals: A comparative, critical review. Annual Review of Psychology, 58, 97-118.

Rescorla, R. A., \& Wagner, A. R. (1972). A theory of Pavlovian conditioning: Variations in the effectiveness of reinforcement and nonreinforcement. In A. H. Black \& W. F. Prokasy (Eds.), Classical conditioning II: Current research and theory (pp. 64-99). New York, NY: Appleton.

Schlottmann, A. (2001). Perception versus knowledge of cause and effect in children: When seeing is believing. Current Directions in Psychological Science, 10, $111-115$.

Schmajuk, N., \& Larrauri, J. (2008). Associative models can describe both causal learning and conditioning. Behavioural Processes, 77, 443-445.

Shanks, D. R. (1985). Forward and backward blocking in human contingency judgment. Quarterly Journal of Experimental Psychology, 37B, 1-21.

Shanks, D. R. (2007). Associationism and cognition: Human contingency learning at 25. Quarterly Journal of Experimental Psychology, 60, 291-309.

Shanks, D. R. (2010). Learning: From association to cognition. Annual Review of Psychology, 61, 273-301.

Simms, V., McCormack, T., \& Beckers, T. (2012). Additivity pretraining and cue competition effects: Developmental evidence for a reasoning-based account of causal learning. Journal of Experimental Psychology: Animal Behavior Processes, 38, 180-190.

Spellman, B. A., Kincannon, A., \& Stose, S. (2005). The relation between counterfactual and causal reasoning. In D. Mandel, D. Hilton, \& P. Catellani (Eds.), The psychology of counterfactual thinking. London, UK: Routledge.

Thompson, V. A., \& Byrne, R. M. J. (2002). Reasoning about things that didn't happen. Journal of 
Experimental Psychology: Learning, Memory, and Cognition, 28, 1154-1170.

Urcelay, G. P., \& Miller, R. R. (2010). Potentiation and overshadowing in Pavlovian fear conditioning. Journal of Experimental Psychology: Animal Behavior Processes, 35, 340-356.

Vandorpe, S., \& De Houwer, J. (2005). A comparison of forward blocking and reduced overshadowing in human causal learning. Psychonomic Bulletin $E$ Revierw, 12, 945-949.
Vandorpe, S., De Houwer, J., \& Beckers, T. (2005). Further evidence for the role of inferential reasoning in forward blocking. Memory \& Cognition, 33, 1047-1056.

Wells, G., \& Gavanski, I. (1989). Mental simulation of causality. Journal of Personality and Social Psychology, 56, 161-169.

Zimmerman, C. (2000). The development of scientific reasoning skills. Developmental Review, 20, 99-149. 